\title{
Bounds for the M-spectral radius of a fourth-order partially symmetric tensor
}

Suhua Li and Yaotang Li*

\section{"Correspondence:}

liyaotang@ynu.edu.cn

School of Mathematics and

Statistics, Yunnan University,

Kunming, Yunnan 650091, P.R. China

\begin{abstract}
M-eigenvalues of fourth-order partially symmetric tensors play an important role in many real fields such as quantum entanglement and nonlinear elastic materials analysis. In this paper, we give two bounds for the maximal absolute value of all the M-eigenvalues (called the M-spectral radius) of a fourth-order partially symmetric tensor and discuss the relation of them. A numerical example is given to explain the proposed results.
\end{abstract}

Keywords: M-eigenvalues; M-spectral radius; partially symmetric; tensors

\section{Introduction}

A fourth-order real tensor $\mathcal{A}=\left(a_{i_{1} i_{2} i_{3} i_{4}}\right) \in \mathbb{R}^{m \times n \times m \times n}$ is called partially symmetric [1] if it has the following symmetry:

$$
a_{i_{1} i_{2} i_{3} i_{4}}=a_{i_{3} i_{2} i_{1} i_{4}}=a_{i_{1} i_{4} i_{3} i_{2}}, \quad \forall i_{1}, i_{3} \in[m], \forall i_{2}, i_{4} \in[n],
$$

where $[m]=\{1,2, \ldots, m\}$ and $[n]=\{1,2, \ldots, n\}$. Such a tensor often arises in nonlinear elastic materials analysis $[2,3]$ and entanglement studies in quantum physics [4-6]. For this tensor, there are many kinds of eigenvalues such as $\mathrm{H}$-eigenvalues, Z-eigenvalues, and Deigenvalues $[7,8]$; here we only discuss its M-eigenvalues [1, 9].

Definition 1 ([9]) Let $\mathcal{A}=\left(a_{i_{1} i_{2} i_{3} i_{4}}\right) \in \mathbb{R}^{m \times n \times m \times n}$ be a partially symmetric tensor, and let $\lambda \in \mathbb{R}$. Suppose that there are real vectors $x \in \mathbb{R}^{m}$ and $y \in \mathbb{R}^{n}$ such that

$$
\left\{\begin{array}{l}
\mathcal{A} \cdot y x y=\lambda x, \\
\mathcal{A} x y x \cdot=\lambda y, \\
x^{T} x=1 \\
y^{T} y=1
\end{array}\right.
$$

where $\mathcal{A} \cdot y x y \in \mathbb{R}^{m}$ and $\mathcal{A} x y x \cdot \in \mathbb{R}^{n}$ with $i$ th components

$$
(\mathcal{A} \cdot y x y)_{i}=\sum_{\left.i_{3} \in[m]\right]_{2}, i_{4} \in[n]} a_{i i_{2} i_{3} i_{4}} y_{i_{2}} x_{i_{3}} y_{i_{4}}
$$

(c) The Author(s) 2018. This article is distributed under the terms of the Creative Commons Attribution 4.0 International License (http://creativecommons.org/licenses/by/4.0/), which permits unrestricted use, distribution, and reproduction in any medium, provided you give appropriate credit to the original author(s) and the source, provide a link to the Creative Commons license, and indicate if changes were made. 
and

$$
(\mathcal{A x y x} \cdot)_{i}=\sum_{i_{1}, i_{3} \in[m]} \sum_{i_{2} \in[n]} a_{i_{1} i_{2} i_{3} i} x_{i_{1}} y_{i_{2}} x_{i_{3}} .
$$

Then $\lambda$ is called an M-eigenvalue of $\mathcal{A}$ with left M-eigenvector $x$ and right M-eigenvector $y$.

Note that M-eigenvalues of a fourth-order partially symmetric tensor always exist [1]. They have a close relation to many problems in the theory of elasticity and quantum physics $[1,9,10]$. For example, the largest M-eigenvalue of $\mathcal{A}=\left(a_{i_{1} i_{2} i_{3} i_{4}}\right) \in \mathbb{R}^{m \times n \times m \times n}$, denoted by

$$
\lambda^{\star}=\max \{\lambda: \lambda \text { is an M-eigenvalue of } \mathcal{A}\},
$$

is the optimum solution of the problem (for details, see [9])

$$
\begin{aligned}
& \max f(x, y)=\sum_{i_{1}, i_{3}=1}^{m} \sum_{i_{2}, i_{4}=1}^{n} a_{i_{1} i_{2} i_{3} i_{4}} x_{i_{1}} y_{i_{2}} x_{i_{3}} y_{i_{4}}, \\
& \text { s.t. } \quad x^{T} x=1, \quad y^{T} y=1, \quad x \in \mathbb{R}^{m}, y \in \mathbb{R}^{n} .
\end{aligned}
$$

The outer product $\lambda x \circ y \circ x \circ y$, where

$$
(\lambda x \circ y \circ x \circ y)_{i_{1} i_{2} i_{3} i_{4}}=\lambda x_{i_{1}} y_{i_{2}} x_{i_{3}} y_{i_{4}}, \quad \forall i_{1}, i_{3} \in[m], \forall i_{2}, i_{4} \in[n]
$$

and $\lambda$ is an M-eigenvalue with the maximal absolute value of $\mathcal{A}=\left(a_{i_{1} i_{2} i_{3} i_{4}}\right) \in \mathbb{R}^{m \times n \times m \times n}$ with left M-eigenvector $x \in \mathbb{R}^{m}$ and right M-eigenvector $y \in \mathbb{R}^{n}$, is a partially symmetric best rank-one approximation of $\mathcal{A}[1]$, which has wide applications in signal and image processing, wireless communication systems, and independent component analysis [1114]. The M-spectral radius of $\mathcal{A}=\left(a_{i_{1} i_{2} i_{3} i_{4}}\right) \in \mathbb{R}^{m \times n \times m \times n}$, denoted by

$$
\rho_{\mathrm{M}}(\mathcal{A})=\max \{|\lambda|: \lambda \text { is an M-eigenvalue of } \mathcal{A}\},
$$

has significant impacts on identifying nonsingular $\mathscr{M}$-tensors, which satisfy the strong ellipticity condition [10].

To our knowledge, there are few results about bounds for the M-spectral radius of a fourth-order partially symmetric tensor. In this paper, we present two bounds for the Mspectral radius and discuss their relation. A numerical example is also given to explain the proposed results.

\section{Two bounds for the $\mathbf{M}$-spectral radius}

In this section, we give two bounds for the M-spectral radius of fourth-order partially symmetric tensors and discuss their relation.

Theorem 1 Let $\mathcal{A}=\left(a_{i_{1} i_{2} i_{3} i_{4}}\right) \in \mathbb{R}^{m \times n \times m \times n}$ be a partially symmetric tensor. Then

$$
\rho_{\mathrm{M}}(\mathcal{A}) \leq \sqrt{\max _{i \in[m]}\left\{R_{i}(\mathcal{A})\right\} \cdot \max _{l \in[n]}\left\{C_{l}(\mathcal{A})\right\}}
$$


where

$$
R_{i}(\mathcal{A})=\sum_{i_{3} \in[m]} \sum_{i_{2}, i_{4} \in[n]}\left|a_{i i_{2} i_{3} i_{4}}\right|, \quad C_{l}(\mathcal{A})=\sum_{i_{1}, i_{3} \in[m]} \sum_{i_{2} \in[n]}\left|a_{i_{1} i_{2} i_{3} l}\right| .
$$

Proof Suppose that $\lambda$ is an M-eigenvalue of $\mathcal{A}$ and that $x \in \mathbb{R}^{m}$ and $y \in \mathbb{R}^{n}$ are associated left M-eigenvector and right $\mathrm{M}$-eigenvector. Then (1) holds. Let

$$
x_{p}=\max _{k \in[m]}\left\{\left|x_{k}\right|\right\}, \quad y_{q}=\max _{k \in[n]}\left\{\left|y_{k}\right|\right\} .
$$

Since $x^{T} x=1$ and $y^{T} y=1$, we have

$$
0<\left|x_{p}\right| \leq 1, \quad 0<\left|y_{q}\right| \leq 1
$$

The $p$ th equation of $\mathcal{A} \cdot y x y=\lambda x$ is

$$
\lambda x_{p}=\sum_{i_{3} \in[m]} \sum_{i_{2}, i_{4} \in[n]} a_{p i_{2} i_{3} i_{4}} y_{i_{2}} x_{i_{3}} y_{i_{4}} .
$$

Taking the absolute values on both sides of (4) and using the triangle inequality give

$$
\begin{aligned}
|\lambda|\left|x_{p}\right| & \leq \sum_{i_{3} \in[m]} \sum_{i_{2}, i_{4} \in[n]}\left|a_{p i_{2} i_{3} i_{4}}\right|\left|y_{i_{2}}\right|\left|x_{i_{3}}\right|\left|y_{i_{4}}\right| \\
& \leq \sum_{i_{3} \in[m]} \sum_{i_{2}, i_{4} \in[n]}\left|a_{p i_{2} i_{3} i_{4}}\right|\left|y_{q}\right| \\
& =R_{p}(\mathcal{A})\left|y_{q}\right| .
\end{aligned}
$$

Similarly, by the $q$ th equation of $\mathcal{A} x y x \cdot=\lambda y$ we have

$$
\begin{aligned}
|\lambda|\left|y_{q}\right| & \leq \sum_{i_{1}, i_{3} \in[m]} \sum_{i_{2} \in[n]}\left|a_{i_{1} i_{2} i_{3} q}\right|\left|x_{i_{1}}\right|\left|y_{i_{2}}\right|\left|x_{i_{3}}\right| \\
& \leq \sum_{i_{1}, i_{3} \in[m]} \sum_{i_{2} \in[n]}\left|a_{i_{1} i_{2} i_{3} q}\right|\left|x_{p}\right| \\
& =C_{q}(\mathcal{A})\left|x_{p}\right| .
\end{aligned}
$$

Multiplying (5) and (6) gives

$$
|\lambda|^{2}\left|x_{p}\right|\left|y_{q}\right| \leq R_{p}(\mathcal{A}) C_{q}(\mathcal{A})\left|x_{p}\right|\left|y_{q}\right|
$$

which, together with (3), yields

$$
|\lambda|^{2} \leq R_{p}(\mathcal{A}) C_{q}(\mathcal{A}) \leq \max _{i \in[m], l \in[n]}\left\{R_{i}(\mathcal{A}) C_{l}(\mathcal{A})\right\}
$$

Since (7) holds for all M-eigenvalues of $\mathcal{A}$, we have

$$
\rho_{\mathrm{M}}(\mathcal{A}) \leq \sqrt{\max _{i \in[m], l \in[n]}\left\{R_{i}(\mathcal{A}) C_{l}(\mathcal{A})\right\}}=\sqrt{\max _{i \in[m]}\left\{R_{i}(\mathcal{A})\right\} \cdot \max _{l \in[n]}\left\{C_{l}(\mathcal{A})\right\}},
$$

and the conclusion follows. 
Theorem 2 Let $\mathcal{A}=\left(a_{i_{1} i_{2} i_{3} i_{4}}\right) \in \mathbb{R}^{m \times n \times m \times n}$ be a partially symmetric tensor, and let $\alpha$ be any subset of $[m]$ and $\beta$ be any subset of $[n]$. Then

$$
\rho_{\mathrm{M}}(\mathcal{A}) \leq \min \left\{\mu_{1}, \mu_{2}\right\}
$$

where

$$
\begin{aligned}
& \mu_{1}=\min _{\alpha \subseteq[m]}\left\{\max _{p \in[m], q \in[n]}\left\{\frac{1}{2}\left(R_{p}^{\alpha}(\mathcal{A})+\sqrt{R_{p}^{\alpha}(\mathcal{A})^{2}+4\left(R_{p}(\mathcal{A})-R_{p}^{\alpha}(\mathcal{A})\right) C_{q}(\mathcal{A})}\right)\right\}\right\}, \\
& \mu_{2}=\min _{\beta \subseteq[n]}\left\{\max _{p \in[m], q \in[n]}\left\{\frac{1}{2}\left(C_{q}^{\beta}(\mathcal{A})+\sqrt{C_{q}^{\beta}(\mathcal{A})^{2}+4\left(C_{q}(\mathcal{A})-C_{q}^{\beta}(\mathcal{A})\right) R_{p}(\mathcal{A})}\right)\right\}\right\},
\end{aligned}
$$

and

$$
R_{p}^{\alpha}(\mathcal{A})=\sum_{i_{3} \in \alpha} \sum_{i_{2}, i_{4} \in[n]}\left|a_{p i_{2} i_{3} i_{4}}\right|, \quad C_{q}^{\beta}(\mathcal{A})=\sum_{i_{2} \in \beta} \sum_{i_{1}, i_{3} \in[m]}\left|a_{i_{1} i_{2} i_{3} q}\right| .
$$

Proof Assume that $\lambda$ is an M-eigenvalue of $\mathcal{A}$ and that $x \in \mathbb{R}^{m}$ and $y \in \mathbb{R}^{n}$ are the corresponding left $\mathrm{M}$-eigenvector and right $\mathrm{M}$-eigenvector. Then (1) holds. Let

$$
\left|x_{p}\right|=\max _{k \in[m]}\left\{\left|x_{k}\right|\right\}, \quad\left|y_{q}\right|=\max _{k \in[n]}\left\{\left|y_{k}\right|\right\} .
$$

Then (3) holds. The $p$ th equation of $\mathcal{A} \cdot y x y=\lambda x$ can be rewritten as

$$
\lambda x_{p}=\sum_{i_{3} \in \alpha} \sum_{i_{2}, i_{4} \in[n]} a_{p i_{2} i_{3} i_{4}} y_{i_{2}} x_{i_{3}} y_{i_{4}}+\sum_{i_{3} \notin \alpha} \sum_{i_{2}, i_{4} \in[n]} a_{p i_{2} i_{3} i_{4}} y_{i_{2}} x_{i_{3}} y_{i_{4}} .
$$

By the technique for the inequality in Theorem 1, we obtain from (9) that

$$
\begin{aligned}
|\lambda|\left|x_{p}\right| & \leq \sum_{i_{3} \in \alpha} \sum_{i_{2}, i_{4} \in[n]}\left|a_{p i_{2} i_{3} i_{4}}\right|\left|y_{i_{2}}\right|\left|x_{p}\right|\left|y_{i_{4}}\right|+\sum_{i_{3} \notin \alpha} \sum_{i_{2}, i_{4} \in[n]}\left|a_{p i_{2} i_{3} i_{4}}\right|\left|y_{i_{2}}\right|\left|x_{i_{3}}\right|\left|y_{q}\right| \\
& \leq \sum_{i_{3} \in \alpha} \sum_{i_{2}, i_{4} \in[n]}\left|a_{p i_{2} i_{3} i_{4}}\right|\left|x_{p}\right|+\sum_{i_{3} \notin \alpha} \sum_{i_{2}, i_{4} \in[n]}\left|a_{p i_{2} i_{3} i_{4}}\right|\left|y_{q}\right| \\
& =R_{p}^{\alpha}(\mathcal{A})\left|x_{p}\right|+\left(R_{p}(\mathcal{A})-R_{p}^{\alpha}(\mathcal{A})\right)\left|y_{q}\right|,
\end{aligned}
$$

that is,

$$
\left(|\lambda|-R_{p}^{\alpha}(\mathcal{A})\right)\left|x_{p}\right| \leq\left(R_{p}(\mathcal{A})-R_{p}^{\alpha}(\mathcal{A})\right)\left|y_{q}\right|
$$

In addition, by the $q$ th equation of $\mathcal{A} x y x \cdot=\lambda y$ we have

$$
|\lambda|\left|y_{q}\right| \leq \sum_{i_{1}, i_{3} \in[m]} \sum_{i_{2} \in[n]}\left|a_{i_{1} i_{2} i_{3} q}\right|\left|x_{p}\right|=C_{q}(\mathcal{A})\left|x_{p}\right|
$$

Multiplying (10) with (11) and using (3) yield

$$
\left(|\lambda|-R_{p}^{\alpha}(\mathcal{A})\right)|\lambda| \leq\left(R_{p}(\mathcal{A})-R_{p}^{\alpha}(\mathcal{A})\right) C_{q}(\mathcal{A})
$$


Then

$$
\begin{aligned}
|\lambda| & \leq \frac{1}{2}\left(R_{p}^{\alpha}(\mathcal{A})+\sqrt{R_{p}^{\alpha}(\mathcal{A})^{2}+4\left(R_{p}(\mathcal{A})-R_{p}^{\alpha}(\mathcal{A})\right) C_{q}(\mathcal{A})}\right) \\
& \leq \max _{p \in[m], q \in[n]}\left\{\frac{1}{2}\left(R_{p}^{\alpha}(\mathcal{A})+\sqrt{R_{p}^{\alpha}(\mathcal{A})^{2}+4\left(R_{p}(\mathcal{A})-R_{p}^{\alpha}(\mathcal{A})\right) C_{q}(\mathcal{A})}\right)\right\} .
\end{aligned}
$$

Note that (13) holds for all M-eigenvalues of $\mathcal{A}$ and any $\alpha \subseteq[m]$. Hence

$$
\rho_{\mathrm{M}}(\mathcal{A}) \leq \mu_{1}
$$

On the other hand, for the $q$ th equation of $\mathcal{A} x y x \cdot=\lambda y$, we have

$$
\lambda y_{q}=\sum_{i_{2} \in \beta} \sum_{i_{1}, i_{3} \in[m]} a_{i_{1} i_{2} i_{3} q} x_{i_{1}} y_{i_{2}} x_{i_{3}}+\sum_{i_{2} \notin \beta} \sum_{i_{1}, i_{3} \in[m]} a_{i_{1} i_{2} i_{3} q} x_{i_{1}} y_{i_{2}} x_{i_{3}} .
$$

Then

$$
\begin{aligned}
|\lambda|\left|y_{q}\right| & \leq \sum_{i_{2} \in \beta} \sum_{i_{1}, i_{3} \in[m]}\left|a_{i_{1} i_{2} i_{3} q}\right|\left|y_{q}\right|+\sum_{i_{2} \notin \beta} \sum_{i_{1}, i_{3} \in[m]}\left|a_{i_{1} i_{2} i_{3} q}\right|\left|x_{p}\right| \\
& =C_{q}^{\beta}(\mathcal{A})\left|y_{q}\right|+\left(C_{q}(\mathcal{A})-C_{q}^{\beta}(\mathcal{A})\right)\left|x_{p}\right|
\end{aligned}
$$

that is,

$$
\left(|\lambda|-C_{q}^{\beta}(\mathcal{A})\right)\left|y_{q}\right| \leq\left(C_{q}(\mathcal{A})-C_{q}^{\beta}(\mathcal{A})\right)\left|x_{p}\right|
$$

By the $p$ th equation of $\mathcal{A} \cdot y x y=\lambda x$ we have

$$
|\lambda|\left|x_{p}\right| \leq \sum_{i_{3} \in[m]} \sum_{i_{2}, i_{4} \in[n]}\left|a_{p i_{2} i_{3} i_{4}}\right|\left|y_{q}\right|=R_{p}(\mathcal{A})\left|y_{q}\right|
$$

Multiplying (16) with (17) and using (3), we derive

$$
\left(|\lambda|-C_{q}^{\beta}(\mathcal{A})\right)|\lambda| \leq\left(C_{q}(\mathcal{A})-C_{q}^{\beta}(\mathcal{A})\right) R_{p}(\mathcal{A}) .
$$

Hence

$$
\begin{aligned}
|\lambda| & \leq \frac{1}{2}\left(C_{q}^{\beta}(\mathcal{A})+\sqrt{C_{q}^{\beta}(\mathcal{A})^{2}+4\left(C_{q}(\mathcal{A})-C_{q}^{\beta}(\mathcal{A})\right) R_{p}(\mathcal{A})}\right) \\
& \leq \max _{p \in[m], q \in[n]}\left\{\frac{1}{2}\left(C_{q}^{\beta}(\mathcal{A})+\sqrt{C_{q}^{\beta}(\mathcal{A})^{2}+4\left(C_{q}(\mathcal{A})-C_{q}^{\beta}(\mathcal{A})\right) R_{p}(\mathcal{A})}\right)\right\} .
\end{aligned}
$$

Since (19) holds for all M-eigenvalues of $\mathcal{A}$ and any $\beta \subseteq[n]$, we have

$$
\rho_{\mathrm{M}}(\mathcal{A}) \leq \mu_{2}
$$

From (14) and (20) we have

$$
\rho_{\mathrm{M}}(\mathcal{A}) \leq \min \left\{\mu_{1}, \mu_{2}\right\} .
$$

The proof is completed. 
Remark 1 Since

$$
\begin{aligned}
& \max _{p \in[m], q \in[n]}\left\{\frac{1}{2}\left(R_{p}^{\alpha}(\mathcal{A})+\sqrt{R_{p}^{\alpha}(\mathcal{A})^{2}+4\left(R_{p}(\mathcal{A})-R_{p}^{\alpha}(\mathcal{A})\right) C_{q}(\mathcal{A})}\right)\right\} \\
& =\max _{p \in[m], q \in[n]}\left\{\frac{1}{2}\left(C_{q}^{\beta}(\mathcal{A})+\sqrt{C_{q}^{\beta}(\mathcal{A})^{2}+4\left(C_{q}(\mathcal{A})-C_{q}^{\beta}(\mathcal{A})\right) R_{p}(\mathcal{A})}\right)\right\} \\
& =\sqrt{\max _{p \in[m]}\left\{R_{p}(\mathcal{A})\right\} \cdot \max _{q \in[n]}\left\{C_{q}(\mathcal{A})\right\}}
\end{aligned}
$$

when $\alpha=\varnothing$ and $\beta=\varnothing$, we have

$$
\min \left\{\mu_{1}, \mu_{2}\right\} \leq \sqrt{\max _{p \in[m]}\left\{R_{p}(\mathcal{A})\right\} \cdot \max _{q \in[n]}\left\{C_{q}(\mathcal{A})\right\}}
$$

Therefore, the bound in (8) is tighter than the bound in (2) for the M-spectral radius $\rho_{\mathrm{M}}(\mathcal{A})$ of a given tensor $\mathcal{A}$.

Remark 2 Although the bound in (8) is tighter than the bound in (2), it is easier to compute the bound in (2) for the $\mathrm{M}$-spectral radius of a given tensor.

Next, we use a numerical example to show the effectiveness of the bounds in Theorems 1 and 2 .

Example 1 Consider the partially symmetric tensor $\mathcal{A}_{1}=\left(a_{i_{1} i_{2} i_{3} i_{4}}\right) \in \mathbb{R}^{3 \times 3 \times 3 \times 3}$ with

$$
\begin{aligned}
& a_{1111}=1.1112, \quad a_{1311}=6.1096, \quad a_{3111}=0.3032, \quad a_{2121}=1.4125, \\
& a_{3131}=1, \quad a_{1212}=0.0788, \quad a_{2222}=1, \quad a_{3222}=0.6032, \\
& a_{3232}=0.3657, \quad a_{1313}=2, \quad a_{2323}=0.6226, \quad a_{3333}=0.3,
\end{aligned}
$$

and the remaining zero elements. By Theorem 1 we have

$$
\rho_{\mathrm{M}}\left(\mathcal{A}_{1}\right) \leq 12.6843
$$

By Theorem 2 we have

$$
\rho_{\mathrm{M}}\left(\mathcal{A}_{1}\right) \leq 10.2397
$$

In fact, $\rho_{\mathrm{M}}\left(\mathcal{A}_{1}\right) \approx 7.6841$

\section{Conclusions}

In this paper, we have presented two bounds for the M-spectral radius of a fourth-order partially symmetric tensor and have indicated their relation. To show the effectiveness of the proposed results, a numerical example is also given. 
Competing interests

The authors declare that they have no competing interests.

\section{Authors' contributions}

Both authors contributed equally to this work. Both authors read and approved the final manuscript.

\section{Publisher's Note}

Springer Nature remains neutral with regard to jurisdictional claims in published maps and institutional affiliations.

Received: 25 October 2017 Accepted: 2 January 2018 Published online: 12 January 2018

\section{References}

1. Qi, LQ, Dai, HH, Han, DR: Conditions for strong ellipticity and M-eigenvalues. Front. Math. China 4(2), 349-364 (2009)

2. Walton, JR, Wilber, JP: Sufficient conditions for strong ellipticity for a class of anisotropic materials. Int. J. Non-Linear Mech. 38(4), 411-455 (2003)

3. Chirita, S, Danescu, A, Ciarletta, M: On the strong ellipticity of the anisotropic linearly elastic materials. J. Elast. 87(1), 1-27 (2007)

4. Dahl, G, Leinaas, JM, Myrheim, J, Ovrum, E: A tensor product matrix approximation problem in quantum physics. Linear Algebra Appl. 420(2-3), 711-725 (2007)

5. Doherty, AC, Parillo, PA, Spedalieri, FM: Distinguishing separable and entangled states. Phys. Rev. Lett. 88(18), Article ID 187904 (2002)

6. Gurvits, L: Classical deterministic complexity of Edmonds' problem and quantum entanglement. In: Proceedings of the Thirty-Fifth ACM Symposium on Theory of Computing, pp. 10-19. ACM, New York (2003)

7. Qi, LQ: Eigenvalues of a real supersymmetric tensor. J. Symb. Comput. 40(6), 1302-1324 (2005)

8. Qi, LQ, Wang, YJ, Wu, EX: D-eigenvalues of diffusion kurtosis tensors. J. Comput. Appl. Math. 221(1), 150-157 (2008)

9. Wang, YJ, Qi, LQ, Zhang, XZ: A practical method for computing the largest M-eigenvalue of a fourth-order partially symmetric tensor. Numer. Linear Algebra Appl. 16(7), 589-601 (2009)

10. Ding, WY, Liu, JJ, Qi, LQ, Yan, H: Elasticity $\mathscr{M}$-tensors and the strong ellipticity condition. arXiv:1705.09911

11. Cardoso, JF: High-order contrasts for independent component analysis. Neural Comput. 11, 157-192 (1999)

12. Lathauwer, $\mathrm{LD}, \mathrm{Moor}, \mathrm{BD}$, Vandewalle, J: On the best rank-1 and rank- $\left(R_{1}, R_{2}, \ldots, R_{N}\right)$ approximation of higher-order tensors. SIAM J. Matrix Anal. Appl. 21(4), 1324-1342 (2000)

13. Kofidis, E, Regalia, PA: On the best rank-1 approximation of higher-order supersymmetric tensors. SIAM J. Matrix Anal. Appl. 23(3), 863-884 (2002)

14. Qi, LQ, Wang, F, Wang, YJ: Z-eigenvalue methods for a global polynomial optimization problem. Math. Program. 118 301-316 (2009)

\section{Submit your manuscript to a SpringerOpen ${ }^{\circ}$ journal and benefit from:}

- Convenient online submission

- Rigorous peer review

- Open access: articles freely available online

- High visibility within the field

- Retaining the copyright to your article

Submit your next manuscript at $\gg$ springeropen.com 